\title{
Psoriasiform pemphigus foliaceus: a report of two cases
}

Pemphigus foliaceus $(\mathrm{PF})$ represents an autoimmune blistering disease characterized by the disruption of epidermal intercellular adhesion proteins. Clinical findings include superficial crusted erosions in a seborrheic distribution; however, the disease can rarely present as an exfoliative erythroderma. Histopathologic findings include acantholysis with cleavage within the granular layer. Direct immunofluorescence studies show intercellular IgG and complement deposition. We present two patients, to our dermatology department, with a previous diagnosis of psoriasis, with an exfoliative erythroderma, which ultimately proved to be because of PF based on histopathological features, direct immunofluorescence results and levels of antibodies against desmoglein 1. Both patients responded well to oral prednisone and rituximab. This variant of $\mathrm{PF}$ should be entertained in both the clinical differential diagnosis of psoriasiform erythroderma and in the microscopic differential diagnosis of psoriasiform epidermal hyperplasia with focal acantholysis, particularly in patients for whom the clinical history is not classic for psoriasis or for whom conventional psoriasis therapies have not proven beneficial.

Keywords: dermatopathology, pemphigus, psoriasis

Grekin SJ, Fox MC, Gudjonsson JE, Fullen DR. Psoriasiform pemphigus foliaceus: a report of two cases.

J Gutan Pathol 2012; 39: 549-553. (C) 2012 John Wiley \& Sons A/S.

\section{Sarah J. Grekin ${ }^{1}$, Matthew C. Fox ${ }^{1}$, Johann E. Gudjonsson ${ }^{1}$ and Douglas R. Fullen ${ }^{1,2}$}

${ }^{1}$ Department of Dermatology, University of Michigan, Ann Arbor, MI, USA and

${ }^{2}$ Department of Pathology, University of Michigan, Ann Arbor, MI, USA

Douglas R. Fullen, MD,

Department of Pathology, M3261, Medical

Science I, University of Michigan, 1301

Catherine, Ann Arbor, Ml 48109-5602, USA

Tel: +17347644460

Fax: +17347644690

e-mail:dfullen@umich.edu

Accepted for publication November 1, 2011
Pemphigus foliaceus (PF) is an autoimmune blistering disease characterized by the disruption of epidermal intercellular adhesion proteins, including the cadherin desmoglein (DSG) 1. ${ }^{1}$ As PF characteristically manifests with superficial crusted erosions in a seborrheic distribution, the disease can rarely present as an exfoliative erythroderma. ${ }^{1}$

Herein, we present two patients with a previous diagnosis of psoriasis who presented to our dermatology department with an exfoliative erythroderma, which ultimately proved to be because of PF based on histopathological features, direct immunofluorescence results and levels of antibodies against DSG 1 .

\section{Reports of cases}

Patient 1

A 71-year-old African-American man with a history notable for biopsy-proven psoriasis presented to the Emergency Department with near-total body erythroderma. He stated that his skin was flaring. He complained of significant pruritus with scaling and malodor. He also endorsed that he had recently been taking mycophenolate mofetil, although he felt this had worsened his skin condition and therefore discontinued the medication.

The patient appeared well and was in no acute distress. Examining his skin revealed a diffuse erythroderma with significant desquamation and scaling, and superficial erosions of the palms bilaterally (Fig. 1). The erythema and desquamation covered $90 \%$ of his total body surface area.

A punch biopsy revealed psoriasiform epidermal hyperplasia with mild spongiosis, papillomatosis, hypergranulosis and confluent parakeratosis. Subtle acantholysis was present focally within the granular layer of the epidermis (Fig. 2A,B). A superficial perivascular infiltrate of lymphocytes with 


\section{Grekin et al.}

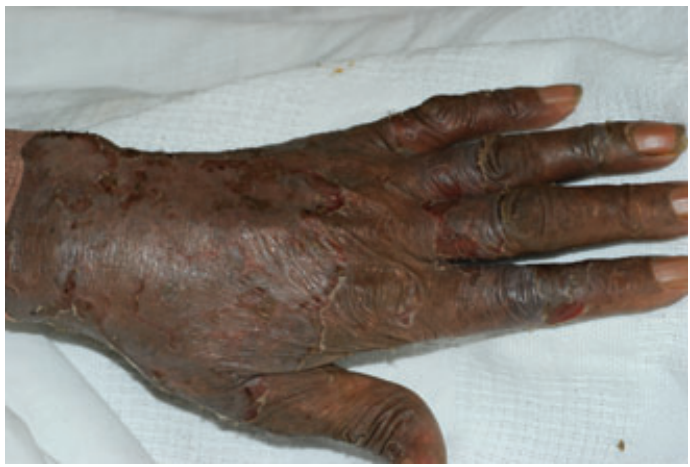

Fig. 1. Desquamation and scaling with superficial erosions involve the hand.

many plasma cells, a few eosinophils and scattered melanophages was observed in the dermis. Direct immunofluorescence examination of the skin biopsy specimen revealed intercellular space binding of granular immunoglobulin $\mathrm{g}$ (IgG), focal granular C3 and weak dermal epidermal junction (DEJ) binding of linear fibrin, consistent with the pemphigus group (Fig. 3). A retrospective review of this patient's biopsy from 1996 revealed psoriasiform epidermal hyperplasia, subcorneal neutrophils and a small focus of superficial acantholysis (Fig. 4). Anti-DSG $1 \mathrm{IgG}$ was elevated to $228.6(<14.0)$, while anti-DSG 3 IgG was normal at $0.33(<9.0)$.

The patient was started on oral prednisone $80 \mathrm{mg}$ daily, but he was unable to tolerate this dose due to insomnia. Therefore, 1 week later his dose was decreased to $60 \mathrm{mg}$ daily. He received two infusions of rituximab 2 weeks apart and showed significant clinical improvement with almost complete clearing of his erythroderma and healing of all areas of desquamation (Fig. 5). At a follow-up appointment 2 weeks after discharge, the patient was satisfied with his improvement and had no appreciable pruritus. A slow taper of prednisone was initiated.

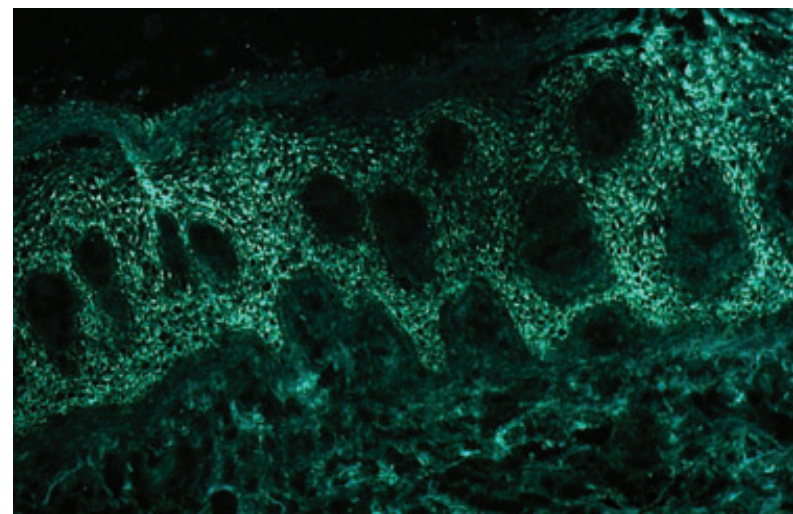

Fig. 3. A biopsy from left abdomen stained with anti-IgG revealed intercellular space binding of granular IgG, consistent with pemphigus (direct immunofluorescence).

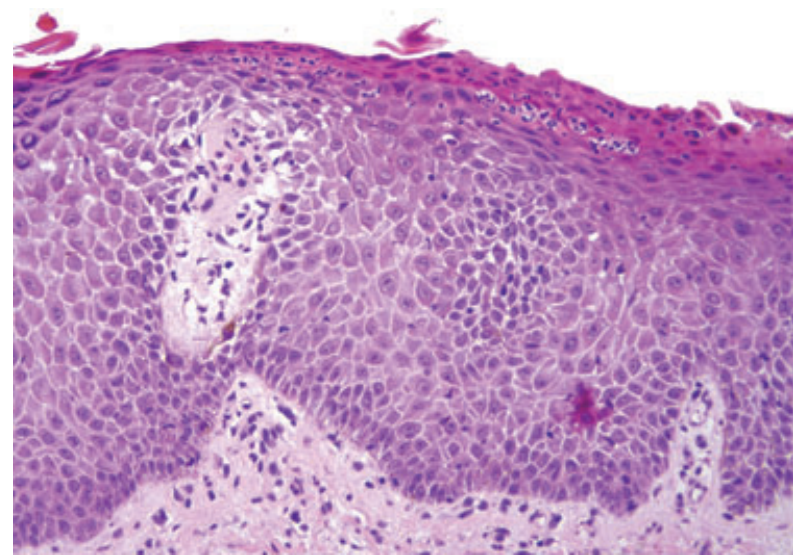

Fig. 4. A biopsy from the same patient, obtained in 1996, showed psoriasiform epidermal hyperplasia, subcorneal neutrophils and a small focus of superficial acantholysis (hematoxylin/eosin).

\section{Patient 2}

A 74-year-old woman with a 3 to 4-year history of psoriasis, confirmed with a biopsy performed by her referring dermatologist, presented to our outpatient
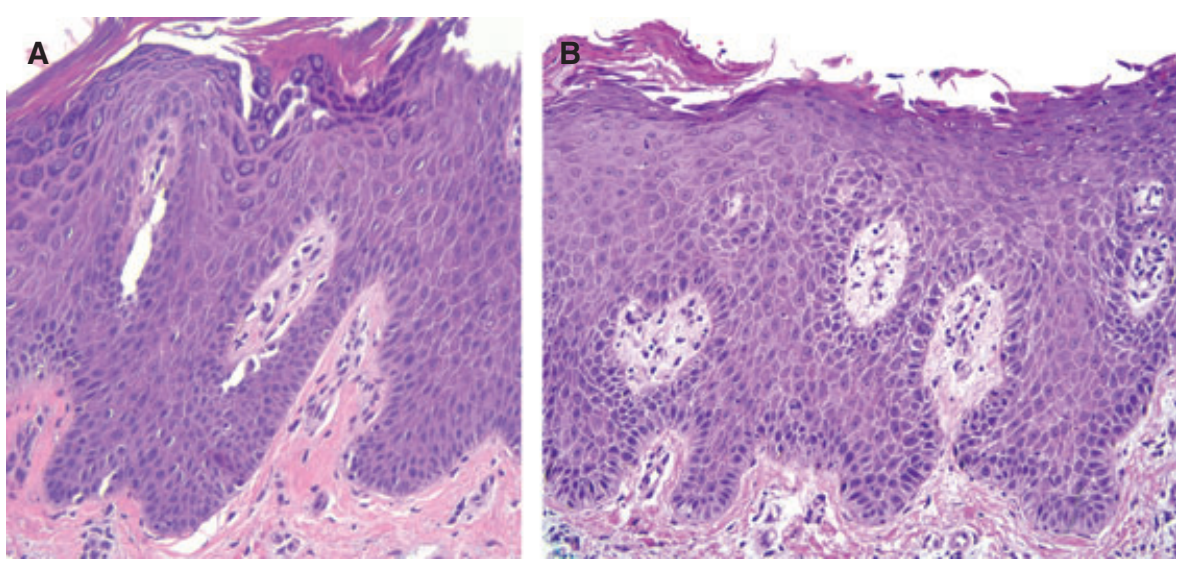

Fig. 2. (A and B) A biopsy from the left abdomen shows psoriasiform epidermal hyperplasia with subtle acantholysis present focally within the granular layer of the epidermis (hematoxylin/eosin). 


\section{Psoriasiform pemphigus foliaceus}

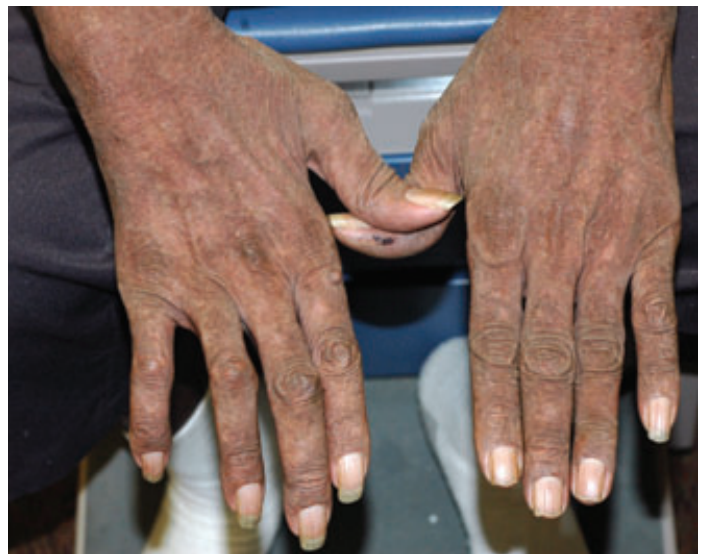

Fig. 5. Status post two infusions of rituximab, 2 weeks apart, with significant clinical improvement and almost complete healing of all areas of desquamation.

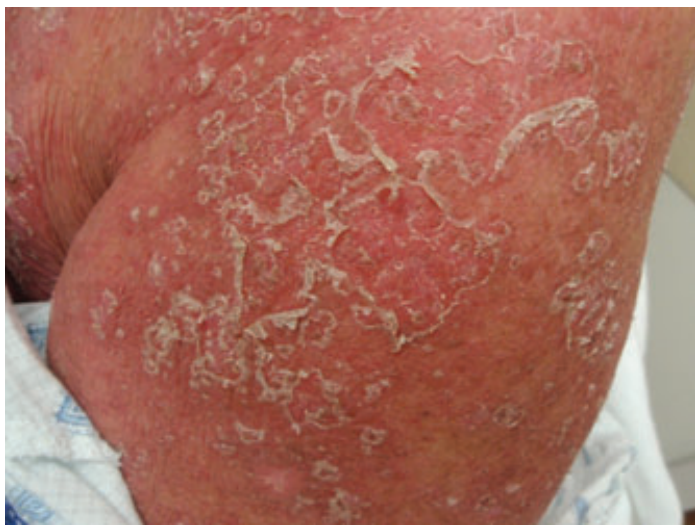

Fig. 6. Near-confluent erythema on the upper arm with associated thick, flake-like scale.

clinic with a brightly erythematous, painful, scaly eruption involving her face, upper trunk, proximal upper extremities and intertriginous folds. Prior to her presentation, her psoriasis had been generally well controlled with narrow band ultraviolet $\mathrm{B}$ (UVB) phototherapy thrice a week. However, recent treatment near home with broadband UVB phototherapy failed to adequately control her disease. At the time of her presentation, she complained of significant erythema, pain and malodorous drainage.

The patient was a well-appearing woman in no acute distress. A total body skin examination showed near-confluent erythema on the face, chest and upper arms with associated thick, flake-like scale (Fig. 6). Macerated erythematous plaques with focal erosions and foul-smelling drainage involved the inframammary and crural folds and axillae.

A punch biopsy showed a small intracorneal pustule with a split near the granular layer and underlying superficial dermal inflammation with neutrophils and eosinophils (Fig. 7A,B). Direct


Fig. 7. (A and B) A biopsy shows an intracorneal pustule with a split near the granular layer and underlying superficial dermal inflammation with neutrophils and eosinophils (hematoxylin/eosin).

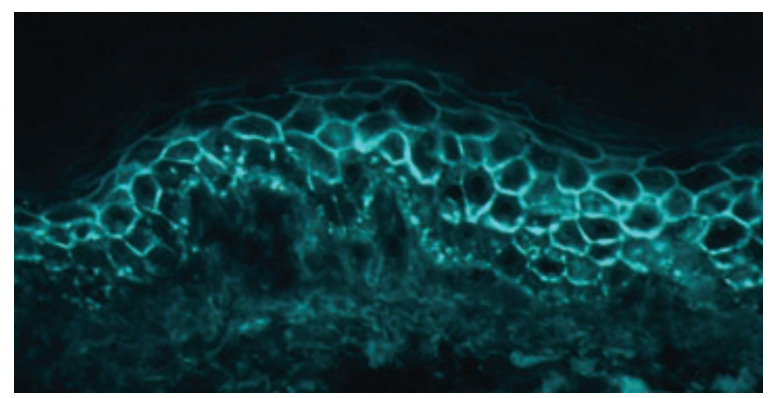

Fig. 8. A biopsy stained with anti-human IgG shows intercellular space deposition of $\operatorname{IgG}$ consistent with pemphigus (direct immunofluorescence).

immunofluorescence examination of the biopsy sample showed intercellular space deposition of IgG consistent with the pemphigus group (Fig. 8). IgG antibodies directed against DSG 1 was elevated to $221.0(<14.0)$, while anti-DSG $3 \mathrm{IgG}$ was normal at $1.2(<9.0)$.

The patient was treated with prednisone at a dose of $1 \mathrm{mg} / \mathrm{kg}$ in combination with mycophenolate mofetil, and topical steroids under occlusion, resulting in rapid improvement of her skin disease. However, several weeks thereafter, she developed thrombocytopenia and acute bacterial cystitis with 


\section{Grekin et al.}

concern for pyelonephritis. Mycophenolate mofetil was discontinued, and the patient was admitted to the hospital for treatment of her bladder infection. Her pemphigus subsequently flared, necessitating an increase in her systemic corticosteroid dose. She continued to flare each time her prednisone dose was decreased, even with slow tapers. Ultimately, the decision was made to initiate treatment with intravenous immunoglobulin and rituximab. Following the second rituximab infusion, her pemphigus has remained well controlled, even with tapering of corticosteroids.

\section{Discussion}

$\mathrm{PF}$ is an uncommon blistering disease. The incidence of $\mathrm{PF}$ varies between populations and has been described in all races. The prevalence of $\mathrm{PF}$ is roughly equal between men and women, and the onset of disease is most common in middle age. ${ }^{2}$

Clinical lesions consist of superficial crusted erosions on an erythematous base, generally confined to the seborrheic areas. ${ }^{2} \mathrm{PF}$ may also present with erythema and scaling localized to the scalp, and this may mimic seborrheic dermatitis., ${ }^{3,4}$ Patients often complain of pain, burning or pruritus. Unlike patients with pemphigus vulgaris (PV), patients with PF rarely develop mucosal lesions. The erosions may become generalized, and occasionally an exfoliative erythroderma may develop. ${ }^{5-10} \mathrm{PF}$ presenting as an exfoliative erythroderma has been reported more frequently in the endemic form of PF, fogo selvagem, ${ }^{1}$ although a case reported by Nousari et al. ${ }^{6}$ describes a patient with non-endemic erythrodermic $\mathrm{PF}$, ultimately proving to be fatal. This case emphasizes the importance of obtaining a specimen for direct immunoflourescence when examining erythrodermic patients. ${ }^{6}$ In a review of 90 cases by Pal et al., ${ }^{8} \mathrm{PF}$ was estimated to cause erythroderma in approximately $6 \%$ of cases.

Histopathologic findings of PF include acantholysis within the granular layer, and because of this, the stratum corneum may be absent. Subcorneal pustules may also be seen. The epidermis deep to the granular layer is usually intact. ${ }^{5}$ Direct immunofluorescence studies show intercellular IgG and complement deposition. ${ }^{2}$

$\mathrm{PF}$ is characterized by $\mathrm{IgG}$ antibodies directed against an epidermal desmosomal protein, specifically DSG $1 .^{1}$ DSG 1 is a cadherin protein found at the desmosomal cell junction. Binding of IgG antibodies to DSG 1 causes acantholysis of keratinocytes in and around the granular layer, where DSG 1 is primarily localized and is critical for cell-to-cell adhesion. $^{2}$ Unlike PV, mucosal surfaces are not involved in PF because mucous membranes primarily express DSG 3, a target in PV, but not PF. ${ }^{2}$

$\mathrm{PF}$ generally follows a chronic course, although spontaneous resolution may occur. Prior to treatment with glucocorticoids, PF was fatal in approximately $60 \%$ of patients. ${ }^{1}$ Therapy is aimed at halting the production of pathogenic antibodies. Therapy varies depending on the severity of disease, but includes treatment with topical steroids for localized disease. Patients with more widespread involvement or erythroderma may be treated with oral steroids in conjunction with various steroidsparing agents such as mycophenolate mofetil or cyclophosphamide. Alternative treatment modalities for patients resistant to more conventional treatments include rituximab, intravenous immunoglobulin or plasmapheresis. ${ }^{5}$

Our two cases are interesting in that the patients had a prior history of psoriasis that had become unresponsive to conventional therapy, and ultimately were diagnosed with PF. Very few patients with both $\mathrm{PF}$ and psoriasis have been reported. ${ }^{1-14} \mathrm{~A}$ few theories have been proposed to explain a potential link between psoriasis and pemphigus. Tomasini et al., ${ }^{13}$ suggested that antigenic stimulation could induce abnormally high levels of IgG against keratinocytes, leading to the development of a bullous disorder. Another theory hypothesizes that inflammation from other skin diseases could create a favorable environment for the expression of pemphigus antigens and improve the activity of pathogenic autoantibodies. ${ }^{12}$ It has also been suggested that altered regulation of $\mathrm{T}$-cells with rising production of autoantibodies may be a conceivable link between the clinical manifestations of these two diseases in the same patient. ${ }^{12}$ Finally, plasminogen activation is known to play a role in acantholysis, and elevated levels of plasminogen activator have been showed in psoriatic lesions. ${ }^{11}$

In our cases, the patients had previous histopathologic diagnoses of psoriasis and had been treated for many years. Interestingly, in a retrospective review of our first patient's initial biopsy, psoriasiform epidermal hyperplasia was noted, but with a small focus of superficial acantholysis. It may be the case that this patient did not in fact have psoriasis, but rather only a psoriasiform variant of PF. This would explain why this patient had been previously unresponsive to conventional psoriasis treatments. In light of her advanced age at the time of diagnosis, it is also possible that our second patient did not have psoriasis, but rather a similar psoriasiform variant of PF. However, this is speculative as the patient's outside records were not available for review, and she may indeed 
Psoriasiform pemphigus foliaceus

have had both psoriasis and PF concomitantly. We believe it is also important to consider a diagnosis of pityriasis rubra pilaris (PRP) when evaluating a patient with generalized erythroderma. PRP can be a difficult diagnosis to make histopathologically, as the microscopic findings may not only be subtle, but can also mimic other skin diseases. In addition, subcorneal acantholysis has been reported in biopsies from patients with PRP resembling PF. ${ }^{15,16}$
This rare variant of $\mathrm{PF}$ should be entertained in the clinical differential diagnosis of erythroderma or a diffuse papulosquamous eruption. It should also be considered in the histopathologic differential diagnosis of psoriasiform epidermal hyperplasia with focal acantholysis, particularly in patients for whom the clinical history is not classic for psoriasis, or for whom conventional psoriasis therapies have not proven beneficial.

\section{References}

1. Connelly EA, Aber C, Kleiner G, Nousari C, Charles C, Schachner LA. Generalized erythrodermic pemphigus foliaceus in a child and its successful response to rituximab treatment. Pediatr Dermatol 2007; 24: 172.

2. Groves RW. Pemphigus: a brief review. Clin Med 2009; 9: 371

3. Jones SK, Schwab HP, Norris DA. Childhood pemphigus foliaceus: case report and review of the literature. Pediatr Dermatol 1986; 3: 459.

4. Chen S, Lu X, Zhou G. Mild pemphigus foliaceus responding to combination therapy with niacinamide and tetracycline. Int J Dermato 2003; 42: 981 .

5. Khachemoune A, Guldbakke KK, Ehrsam E. Pemphigus foliaceus: a case report and short review. Cutis 2006; 78: 105.

6. Nousari HC, Moresi M, Klapper M, Anhalt GJ. Nonendemic pemphigus foliaceus presenting as fatal bullous erythroderma. Cutis 2001; 67: 251
7. Miyakura T, Yamamoto T, Okubo Y, et al Pemphigus foliaceus with prominent neutrophilic pustules initially presenting as erythroderma. Clin Exp Dermatol 2009; 34: e46.

8. Pal S, Haroon TS. Erythroderma: a clinicoetiologic study of 90 cases. Int J Dermatol 1998; 37: 104

9. Grant-Kels JM, Bernstein ML, Rothe MJ Exfoliative dermatitis. In Wolff K, Goldsmith LA, Katz SI, Gilchrest BA, Paller AS, Leffell DJ, eds. Fitzpatrick's dermatology in general medicine, 7 th ed. The McGraw-Hill Companies, 2008; 230

10. Sterry W, Assaf C. Erythroderma. In Bolognia JL, Jorizzo JL, Rapini RP, eds. Dermatology,2nd ed. Elsevier Limited, 2008; 150.

11. Giomi B, Cardinali C, Pestelli E, Caproni M, Fabbri P. Pemphigus foliaceus developing on pre-existing psoriasis: a supposed pathogenic linkage. Acta Derm Venereol 2004; 84: 82.
12. Panzarella K, Camisa C. Coexistence of superficial pemphigus and psoriasis. Cutis 1996; $57: 414$.

13. Tomasini D, Cerri A, Cozzani E, Berti E. Development of pemphigus foliaceus in a patient with psoriasis: a simple coincidence? Eur J Dermatol 1998; 8: 56.

14. Peterson JD, Worobec SM, Chan LS. An erythrodermic variant of pemphigus foliaceus with puzzling histologic and immunopathologic features. J Cutan Med Surg 2007; 11: 179

15. Howe K, Foresman P, Griffin T, Johnson W. Pityriasis rubra pilaris with acantholysis. J Cutan Pathol 1996; 23: 270.

16. Sebastian A, Koff AB, Goldber LJ. PRP with subcorneal acantholysis: case report and review. J Cutan Pathol 2010; 37: 99. 